\title{
Analysis of the Correlation between Macro-economy and Stock Market Volatility Employed by Time-varying Copula and ICA
}

\author{
C.Z. SUN \& Y.W. YANG \\ School of management, Northwestern Polytechnical University, Xi'an Shaanxi, China
}

\begin{abstract}
This paper aims to estimate the dependence between macro-economy and stock market volatility. Firstly, we employ independent component analysis (ICA) to excavate the independent driven factors of the macro-economy and name all the independent components. Secondly, we assume that all margins are submitted to skewed $t$ distribution, and then make use of AR(1)-GJR(1,1)-Skewed t model to fit monthly time series data. Moreover, time-varying normal copula and time-varying SJC copula are used to fit the dependence between macro-economy and stock market volatility and they all behave well. Results show that import and export factor (IC1) can exert a big impact to stock market and the dependence between them varying rapidly through time. The dependence fluctuates great during the appreciation of the RMB against the U.S.
\end{abstract}

KEYWORD: Macro-economy; Stock market; Time-varying Copula; ICA

\section{INTRODUCTION}

The dependence between macro-economy and stock market volatility is one of the most classic problems in financial research. Especially the global economy has suffered the serious consequences of the financial crisis in 2008. To excavate the inner structure and recognize the regulation between macro-economy and stock market volatility appears very important. Officer (1973) found that in the economic depression, the stock market volatility and industrial production and the money supply volatility in macro-economy will go up. Fama (1990) found that the price volatility in stock market mostly suffer from some macroeconomic variables, and lead to the change of macroeconomic indicators and have the function of the "barometer". Beltrattia \& Morana (2006) employ 30 years' S\&P500 data to study the dependence between macro-economy and stock market volatility. They found that stock volatility can also exert some influences to macro-economy volatility, but the latter variable performs stronger. No matter Granger causality test or linear regression model, they all describe linear correlation. However, copula functions can capture not only the linear correlation but also nonlinear correlation. Copula is the functions that join or couple multivariate distribution functions to their one-dimensional marginal distribution functions. Furthermore, timevarying copula functions are more flexible than static copula, and it is more satisfactory to describe dynamic risks. As for data selecting aspect, how to select macroeconomic variable is an issue worthy of further study. The algorithm will be complex and the program will hardly converge if we select too much data. Conversely, the variable can hardly represent the macro-economy. However, the most complex phenomena presented in nonlinear system is driven by a handful of independent factors. Based on the above considerations, we adopt independent component analysis (ICA) to separate the driving factors from multiple observable macroeconomic variables in the macroeconomic system. If we captured these driving factors, it may reflect the essence of macro-economy well.

The rest of the paper is set up in the following manner. Section 2 presents ICA, Mutual Information (MI), GJR (Glosten, Jaganathan \& Runkle, 1993) model and two types of copula functions. Section 3 contains description of data and empirical research. Policy implications are in Section 4, followed by conclusion in the last section.

\section{METHODOLOGY}

\subsection{Independent Component Analysis}

Independent component analysis (ICA) is a statistical and computational technique for revealing hidden factors that underlie sets of random variables, 
measurements, or signals. The condition of its application is that at most one signal is submitted to Gaussian distribution in observed signals, but it is not a serious restriction for financial data. There are further promising applications to risk management since ICA focuses on higher-order statistics.

We use FastICA algorithm to excavate the inner driven factors of macroeconomic volatility. This algorithm is widely used because of its high convergence speed and good separation effect. The specific steps are as follows:

1) The observed signals should be centralized and whitened to get $\mathrm{z}(\mathrm{t})$.

2) Give an initial value to $w_{i} \in R^{n} \mathrm{i}=1,2, \cdots, \mathrm{n}$, and give its norm 1.

3) Refresh $w_{i}$ according to $w_{i} \leftarrow E\left\{z g\left(w_{i}^{T} z\right)\right\}$ $-E\left\{g^{\prime}\left(w_{i}^{T} z\right)\right\} w_{i}$, where $\mathrm{g}$ is a nonlinear function.

4) Orthogonalize matrix $\mathrm{W}: W \leftarrow\left(W W^{T}\right)^{-1 / 2} W$

5) If it does not converge, go back to step 3.

\subsection{Mutual Information}

In Section 3, we will employ mutual information (MI) to name the independent driven factors through ICA. In probability and information theory, the MI of two random variables is a measure of the variables' mutual dependence.

Formally, the MI of two random variables $\mathrm{X}$ and Y can be defined as:

$$
\begin{gathered}
I(x, y)=\sum_{y \in Y} \sum_{x \in X} p(x, y) \log \frac{p(x, y)}{p(x) \mathrm{p}(\mathrm{y})} \\
I(x, y)=\int_{Y} \int_{X} p(x, y) \log \frac{p(x, y)}{p(x) \mathrm{p}(\mathrm{y})} d x d y
\end{gathered}
$$

Equation (1) is in the discrete case, and equation (2) is in the continuous case. Where $p(x, y)$ is the joint distribution (density) function of $\mathrm{X}$ and $\mathrm{Y}$, and $p(x)$ and $\mathrm{p}(\mathrm{y})$ are marginal probability distribution (density) functions of $\mathrm{X}$ and $\mathrm{Y}$ respectively.

\subsection{GJR Model}

Marginal distributions should be determined before modeling copula functions. According to previous studies, economic or financial data have the characteristics of high peak, thick tail, and heteroscedasticity. So this paper select AR(1)GJR(1,1)-Skewed $t$ to describe the marginal distribution of macro-economy and stock market volatility. The specific model is as follows:

$$
r_{i, t}=c_{0}+c_{1} r_{i, t-1}+e_{i, t}
$$

$$
\begin{aligned}
& e_{i, t}=\sqrt{h_{i, t}} \varepsilon_{i, t}, \quad \varepsilon_{i, t} \sim \operatorname{Skewedt}(v, \lambda) \\
& h_{i, t}=w_{i, t}+\alpha e_{i, t-1}^{2}+\beta h_{i, t-1}+\gamma e_{i, t-1}^{2} I_{t-1}\left(e_{i, t-1}<0\right)
\end{aligned}
$$

Where the mean equation (equation 3) is decomposed as one-lagged return $r_{i, t-1}$, conditional mean $c_{0}$ and innovation $e_{i, t} \cdot \varepsilon_{i, t}$ is submitted to skewed $\mathrm{t}$ distribution with parameter $v$ and $\lambda$, and its probability density function is presented in equation 6. $I_{t-1}=1$ if $e_{i, t-1}<0$ and 0 otherwise. If $\gamma>0$, bad news increases volatility, and we say that there is a leverage effect. If $\gamma \neq 0$, the news impact is asymmetric.

$$
\begin{aligned}
& d(z ; v, \lambda)= \\
& \left\{\begin{array}{l}
b c\left[1+\frac{1}{v-2}\left(\frac{b z+a}{1-\lambda}\right)^{2}\right]^{-[(v+1) / 2]} \text { if } z<-a / b \\
b c\left[1+\frac{1}{v-2}\left(\frac{b z+a}{1+\lambda}\right)^{2}\right]^{-[(v+1) / 2]} \text { if } z \geq-a / b
\end{array}\right.
\end{aligned}
$$

Where $\quad a=4 \lambda c \frac{v-2}{v-1}, \quad b^{2}=1+3 \lambda^{2}-a^{2}, \quad c=$ $\frac{\Gamma\left(\frac{v+1}{2}\right)}{\sqrt{\pi(v-2)} \Gamma\left(\frac{v}{2}\right)}$.

where $v$ and $\lambda$ denote the degree-of-freedom parameter and the asymmetry parameter, respectively.

\subsection{Copula Functions}

We adopt two types of time-varying copula to describe the correlation between macro-economy and stock market volatility.

1) Normal Copula

The cumulative distribution function of the bivariate normal copula is as follows:

$C(u, v ; \rho)=$

$\int_{-\infty}^{\phi^{-1}(u)} \int_{-\infty}^{\phi^{-1}(v)} \frac{1}{2 \pi \sqrt{1-\rho^{2}}} \exp \left(\frac{-\left(r^{2}+s^{2}-2 \rho r s\right)}{2\left(1-\rho^{2}\right)}\right) d r d s(7)$

Where the $\rho$ is the Pearson correlation of $\Phi^{-1}(u)$ and $\Phi^{-1}(v)$, both $\mathrm{u}$ and $\mathrm{v}$ are submitted to independent and identically uniform distribution, which are CDFs of the standardized residuals from marginal models. Patton (2001) proposes an $\operatorname{ARMA}(1,10)$ process to describe $\rho_{t}$ :

$$
\rho_{t}=\Lambda\left[\omega_{t}+\beta_{t} \rho_{t-1}+\alpha+\frac{1}{10} \sum_{i=1}^{10} \Phi^{-1}\left(u_{t-i}\right) \Phi^{-1}\left(\mathrm{v}_{t-i}\right)\right]
$$


Where $\Lambda(x)=\frac{1-e^{-x}}{1+e^{-x}}$ is the modified logistic transformation, designed to keep $\rho_{t}$ in $(-1,1)$ at all times.

2) SJC Copula

The cumulative distribution function of the bivariate SJC copula is as follows:

$C_{S J C}\left(u, v ; \tau_{t}^{U}, \tau_{t}^{L}\right)=0.5\left(\mathrm{C}_{J C}\left(u, \mathrm{v} ; \tau_{t}^{U}, \tau_{t}^{L}\right)+C_{J C}(1-u, 1-\right.$ $\left.\left.v ; \tau_{t}^{U}, \tau_{t}^{L}\right)+u+v-1\right)$

Where $\quad C_{J C}\left(u, v ; \tau_{t}^{U}, \tau_{t}^{L}\right)=1-\left(1-\left\{\left[1-(1-\mathrm{u})^{k}\right]^{-\gamma}\right.\right.$ $\left.\left.+\left[1-(1-v)^{k}\right]^{-\gamma}-1\right\}^{-\frac{1}{\gamma}}\right)^{\frac{1}{k}}, \quad k=1 / \log _{2}\left(2-\tau_{t}^{U}\right), \gamma=$ $-1 /\left(\log _{2} \tau_{t}^{L}\right), \tau_{t}^{U}, \tau_{t}^{L}$ are upper tail dependence and lower tail dependence, respectively. Patton (2006) proposes the following evolution equations for the SJC copula:

$$
\tau_{t}^{U}=\Lambda\left[\omega_{U}+\beta_{U} \tau_{t-1}^{U}+\alpha_{U} \times \frac{1}{10} \sum_{i=1}^{10}\left|u_{t-i}-v_{t-i}\right|\right]
$$

$\tau_{t}^{L}=\Lambda\left[\omega_{L}+\beta_{L} \tau_{t-1}^{L}+\alpha_{L} \times \frac{1}{10} \sum_{i=1}^{10}\left|u_{t-i}-v_{t-i}\right|\right]$

Where $\quad \Lambda(x)=\left(1+e^{-x}\right)^{-1}$ is the logistic transformation, used to keep $\tau^{U}$ and $\tau^{L}$ in $(0,1)$ at all times.

\section{DATA AND EMPIRICAL RESULTS}

In this part, we successively exhibit the results of ICA, MI, AR(1)-GJR(1,1)-Skewed $\mathrm{t}$ model and time-varying copula.

\subsection{The Data}

We select 10 Chinese macroeconomic indicators: CPI, PPI, The total amount of import and export, M2 supply, The amount of investment in fixed assets, Industrial added value, National financial revenue, RMB-USD exchange rate, Real estate development and comprehensive economic index and Retail sales of social consumer goods and name them Ii $(i=1,2 \ldots 10)$ (the data come from national bureau of statistics of China and CSMAR). We use monthly data from January 2001 to December 2013. We obtain the log-difference of CPI, PPI and real estate development and comprehensive economic index and the growth rate of the other seven indicators, then normalized. We obtain 4 independent components named ICi $(\mathrm{i}=1,2,3,4)$ from 10 macroeconomic indicators using FastICA algorithm. Table 1 provides the MI (equation 1 and 2) between 4 independent components and 10 macroeconomic indicators. According to MI maximum principal, we name IC1 as import and export factor, IC2 as exchange rate factor, IC3 as investment factor. Although the MI between IC4 and Real estate development and comprehensive economic index reaches 0.7064 , the value between IC4 and other 9 indicators are small and real estate belongs to fixedasset investment, so we name IC4 as noise and exclude it. We obtain Shanghai composite index returns by using log-difference from Shanghai composite index and name it SH.

To comprehend the features of ICs and stock market volatility better, Table 2 provides summary statistics of ICs and SH. As previously found in other studies, all variable demonstrate negative skewness and excess kurtosis. From the results of J$\mathrm{B}$ test, we find that normality hypothesis is significantly rejected. In addition, the $\mathrm{LM}(\mathrm{N})$ statistic tests whether the squared return is serially correlated up to lag N. This statistic clearly indicates that all variables have ARCH effects. So we use GJR model to fit the marginal distribution presented in table 3 .

Table 1. MI between independent components and macroeconomic indicators

\begin{tabular}{|l|c|c|c|c|}
\hline & IC1 & IC2 & IC3 & IC4 \\
\hline CPI & 0.210 & 0.185 & 0.189 & 0.264 \\
\hline PPI & 0.248 & 0.242 & 0.234 & 0.214 \\
\hline The total amount of import and export & 0.418 & 0.257 & 0.388 & 0.215 \\
\hline M2 supply & 0.394 & 0.364 & 0.272 & 0.206 \\
\hline The amount of investment in Fixed assets & 0.339 & 0.329 & 0.617 & 0.183 \\
\hline Industrial added value & 0.311 & 0.255 & 0.365 & 0.212 \\
\hline National financial revenue & 0.166 & 0.196 & 0.204 & 0.166 \\
\hline RMB-USD exchange rate & 0.269 & 0.878 & 0.309 & 0.214 \\
\hline Real estate development and comprehensive economic index & 0.298 & 0.243 & 0.278 & 0.706 \\
\hline Retail sales of social consumer goods & 0.193 & 0.243 & 0.211 & 0.244 \\
\hline
\end{tabular}


Table 2. Data description and statistics

\begin{tabular}{|c|c|c|c|c|}
\hline & IC1 & IC2 & IC3 & SH \\
\hline Mean & $-2.19 \mathrm{E}-09$ & $1.55 \mathrm{E}-10$ & $3.42 \mathrm{E}-10$ & $1.55 \mathrm{E}-02$ \\
\hline Std. Dev. & 1.0032 & 1.0032 & 1.0032 & 8.3006 \\
\hline Skewness & 2.399 & -1.8937 & -1.9893 & -0.4892 \\
\hline Kurtosis & 9.3082 & 7.2031 & 12.4552 & 4.1945 \\
\hline Jarque-Bera & 405.7114 & 206.7354 & 679.6184 & 15.3977 \\
\hline LM(1) & $5.0162 * *$ & $8.9342 * * *$ & $27.9300 * * *$ & 0.0348 \\
\hline LM(5) & $10.51 *$ & $20.30 * * *$ & $36.11 * * *$ & $18.08 * * *$ \\
\hline
\end{tabular}

Notes: In this table as well as all the following ones, coefficients that are significant at the $1 \%, 5 \%$ and $10 \%$ levels are denoted by $* * *, * *$, and $*$, respectively.

\subsection{Results for AR(1)-GJR(1,1)-Skewed t Model}

To appropriate analyze the volatility of the ICs and $\mathrm{SH}$, we use AR(1)-GJR(1,1)-Skewed $\mathrm{t}$ model (equation 3, 4, 5, and 6) to fit the marginal distribution as mentioned earlier. Table 3 shows the results of the model.

The asymmetry parameter $\gamma$ in all groups is negative, and it exhibits that the influence of the information between macro-economy and stock market volatility is asymmetry and good news can generate greater volatility. All groups of $\alpha+\beta+0.5 \gamma$ value is $0.686,0.916,0.852,0.983$, respectively, and it illustrates both macro-economy and stock market has a long-run persistence of volatility.

Table 3. Parameter estimates for the marginal distribution models

\begin{tabular}{|c|c|c|c|c|c|c|c|c|c|}
\hline & $\mathrm{C} 0$ & $\mathrm{C} 1$ & $\omega$ & $\alpha$ & $\beta$ & $\gamma$ & $v$ & $\lambda$ & Log-likelihood \\
\hline \multirow{2}{*}{ IC1 } & -0.116 & 0.50 & 0.083 & 0.50 & 0.312 & -0.25 & 4.401 & -0.102 & \multirow{2}{*}{-95.652} \\
\hline & $(0.459)$ & $(11.479)$ & $(5.018)$ & $(32.423)$ & $(43.78)$ & $(102.615)$ & $(268.594)$ & $(4.287)$ & \\
\hline \multirow{2}{*}{ IC2 } & $-0.162 *$ & $0.50 * * *$ & 0.043 & $0.50 * * *$ & $0.541 * * *$ & -0.25 & $4.179 * * *$ & 0.245 & \multirow{2}{*}{-140.818} \\
\hline & $(0.09)$ & $(0.089)$ & $(0.041)$ & $(0.191)$ & $(0.116)$ & $(0.336)$ & $(1.265)$ & $(0.161)$ & \\
\hline \multirow{2}{*}{ IC3 } & -0.138 & $0.5 * * *$ & $0.069 *$ & $0.5 * * *$ & $0.392 *$ & -0.080 & $4.370 * *$ & $-0.168^{*}$ & \multirow{2}{*}{-104.47} \\
\hline & $(0.1)$ & $(0)$ & $(0.042)$ & $(0.004)$ & $(0.238)$ & $(0.159)$ & $(1.957)$ & $(0.286)$ & \\
\hline \multirow{2}{*}{ SH } & -0.039 & -0.006 & 1.266 & 0.1321 & 0.917 & -0.132 & 99.860 & -0.123 & \multirow{2}{*}{-534.286} \\
\hline & (1.978) & $(0.08)$ & $(12.812)$ & $(0.276)$ & $(0.637)$ & $(0.272)$ & (672.469) & $(0.293)$ & \\
\hline
\end{tabular}

Note: The values in parentheses are standard errors

The asymmetry parameter $\gamma$ in all groups is negative, and it exhibits that the influence of the information between macro-economy and stock market volatility is asymmetry and good news can generate greater volatility. All groups of $\alpha+\beta+0.5 \gamma$ value is $0.686,0.916,0.852,0.983$, respectively, and it illustrates both macro-economy and stock market has a long-run persistence of volatility.

As we know, in a preliminary step to model copula functions, we should obtain marginal distribution uit, and ensure that uit should be i.i.d. Uniform $(0,1)$. If we use a misspecified model for the marginal distributions, then the probability integral transforms will not be Uniform $(0,1)$, and so any copula model will automatically be misspecified[9]. Then we examine the serial correlation of $\left(u_{i t}-\bar{u}_{i}\right)^{k}$, for $\mathrm{k}=2,4$ by regressing $\left(u_{i t}-\bar{u}_{i}\right)^{k}$ on 20 own lags. The LM test statistic is $(\mathrm{T}-20) * \mathrm{R}^{2}$ for each regression and is asymptotically distributed as $\chi_{20}^{2}$ under the null no serial correlation and $\mathrm{R} 2$ is the coefficient of determination of the regression. The p-values from table 4 suggested that serial correlation could not be rejected at the $5 \%$ significance level for all variables.
Therefore, the copula functions could capture the correlation between macro-economy and stock market volatility in the right way.

Table 4. Goodness-of-fit test for marginal distributions

\begin{tabular}{|c|c|c|c|c|}
\hline & \multicolumn{2}{|c|}{ K-S test } & \multicolumn{2}{c|}{ Box-Ljung Test } \\
\hline & statistic & P-value & 2nd moment & 4th moment \\
\hline IC1 & 0.08 & 1 & 0.7818 & 0.4177 \\
\hline IC2 & 0.08 & 1 & 0.113 & 0.427 \\
\hline IC3 & 0.08 & 1 & 0.3727 & 0.2901 \\
\hline SH & 0.08 & 1 & 0.1313 & 0.2247 \\
\hline
\end{tabular}

Note: This table presents the statistics and p-values from K-S tests and p-value from Box-Ljung tests, respectively.

\subsection{Results for Copula Models}

We employ time-varying normal copula and timevarying SJC copula (equation 7 and 9) to model the dependence between macro-economy and stock market volatility as mentioned earlier. According to maximum likelihood estimation, we obtain the estimated parameters of evolution equation (equation 8, 10 and 11) represented in table 5 . 
Table 5. The estimated results of time-varying copula

\begin{tabular}{|c|c|c|c|c|c|c|c|c|c|}
\hline \multicolumn{4}{|c|}{ Normal Copula } & \multicolumn{6}{|c|}{ SJC Copula } \\
\hline & \multirow{2}{*}{ IC1-SH } & \multirow{2}{*}{ IC2-SH } & \multirow{2}{*}{ IC3-SH } & \multicolumn{2}{|c|}{ IC1-SH } & \multicolumn{2}{|c|}{ IC2-SH } & \multicolumn{2}{|c|}{ IC3-SH } \\
\hline & & & & $\lambda^{\mathrm{U}}$ & $\lambda^{\mathrm{L}}$ & $\lambda^{\mathrm{U}}$ & $\lambda^{\mathrm{L}}$ & $\lambda^{\mathrm{U}}$ & $\lambda^{\mathrm{L}}$ \\
\hline$\omega$ & 0.004 & 0.056 & -0.303 & 0.638 & -11.559 & -4.435 & -1.884 & -13.170 & -13.170 \\
\hline$\alpha$ & 0.101 & -0.266 & -0.412 & -9.867 & 25.000 & 1.908 & -2.880 & 0.000 & 0.000 \\
\hline$\beta$ & 1.951 & 2.118 & -1.795 & 1.812 & 3.398 & -0.045 & 0.247 & 0.000 & 0.000 \\
\hline AIC & -12.181 & -14.156 & -1.555 & \multicolumn{2}{|c|}{-16.728} & \multicolumn{2}{|c|}{-4.545} & \multicolumn{2}{|c|}{2.362} \\
\hline BIC & -12.123 & -14.097 & -1.496 & \multicolumn{2}{|c|}{-16.610} & \multicolumn{2}{|c|}{-4.427} & \multicolumn{2}{|c|}{2.480} \\
\hline LL & 6.110 & 7.097 & 0.797 & \multicolumn{2}{|c|}{8.403} & \multicolumn{2}{|c|}{2.311} & \multicolumn{2}{|c|}{-1.143} \\
\hline
\end{tabular}

We take the AIC, BIC as criterions to evaluate the best fit of the different copula models. Time-varying SJC copula is the best fitted model between IC1 (import and export factor) and $\mathrm{SH}$ since the lowest AIC and BIC and time-varying normal copula for IC2 (exchange rate factor), IC3 (investment factor) and SH. Therefore, we obtain the dynamic correlation coefficients from the evolution equation presented in figure 1 and figure 2 .
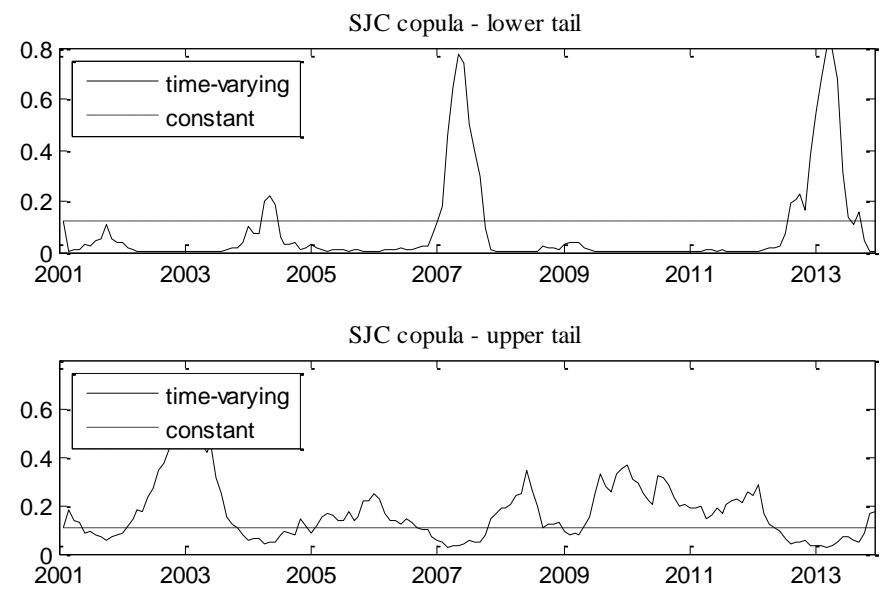

Figure 1. The correlation coefficient between IC1 and SH using time-varying SJC copula
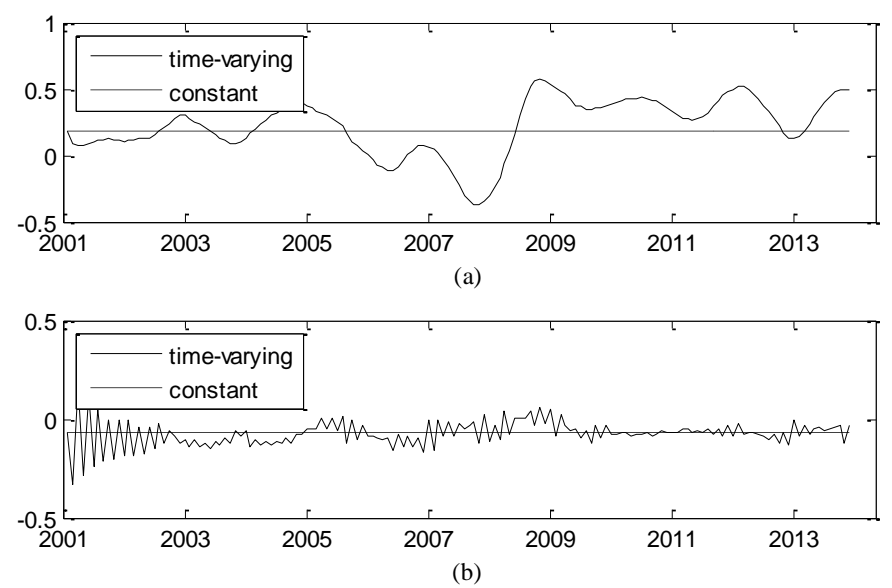

Figure 2. (a) The correlation coefficient between IC2 and SH using time-varying normal copula

(b) The correlation coefficient between IC3 and SH using timevarying normal copula

\section{POLICY IMPLICATION}

Overall, the tail dependence between $\mathrm{IC} 1$ and $\mathrm{SH}$ fluctuates conversely and this conforms to reality. The upper tail correlation between IC1 and $\mathrm{SH}$ increased obviously in the year 2003, it may have the relevance with the General Administration of Quality Supervision, Inspection and Quarantine of the People's Republic of China (AQSIQ)'s taking active steps to encourage import and export and the amount of import and export have reached a peak at this time. During the period 2008, global financial crisis break out and it exerts a huge impact to import and export and lead to the rapidly increase in the lower tail dependence between IC1 and SH. The dependence fluctuates great during the appreciation of the RMB against the U.S. dollar. But the financial crisis leads to economic unrest, and people are averse to invest and hold the cash in spite of the appreciation of the RMB against the USD. In the year 2013, the growth rate of the amount of import and export increased slowly and thus lead to the rapidly increase in the lower tail dependence between IC1 and SH. The correlation between IC3 and $\mathrm{SH}$ has remained stable and small. It may have the relationship with excess capacity of China.

\section{CONCLUSIONS}

In this paper, we employ AR(1)-GJR(1,1)-Skewed t model to fit the marginal distribution for the analysis of volatility of the macro-economy and stock market. Meanwhile, we use the Kolmogorov-Smirnov and Box-Ljung tests to discuss whether the underlying assumptions are satisfied for marginal distribution and the answer is affirmative. In addition, we apply time-varying normal copula and time-varying SJC copula to discuss the dependence between the macro-economy and stock market volatility. According to empirical studies, the normal copula and SJC copula can well depict the correlation between macro-economy and stock market volatility. In addition, the fluctuation of the lower tail dependence between $\mathrm{IC} 1$ and $\mathrm{SH}$ is bigger than its 
upper tail dependence, that is to say, bad news can aggravate the volatility of the dependence between them and exert greater influence than good news. At last, exchange rate factor exerts great influence to stock market volatility. In the short term, the volatility of exchange rate can directly drive the demand and supply of the stock market funds to affect the volatility of stock price. In the long-term run, the government can regulate the stock price through the adjustment of industrial structure and resource configuration.

\section{ACKNOWLEDGEMENTS}

This work was supported by the project from The National Social Science Found of China (No. 13BJY012) and by the Seed Foundation of Innovation and Creation for Graduate Students in Northwestern Polytechnical University (No. Z2015163).

\section{REFERENCES}

[1] Aapo Hyvarinen. et al 2001. Independent component analysis, 1st ed. New York: J. Wiley.

[2] Back, A. \& Weigend, A. 1998. A first application of independent analysis to extracting structure from stock returns, International Journal of Neural Systems (8): 473484.

[3] Beltrattia, A. \& Morana, C. 2006. Breaks and persistency: macroeconomic causes of stock market volatility. Journal of Econometrics 131: 151-177.

[4] Fama E.F. 1990. Stock returns, expected returns and real activity. Journal of Finance (45): 1089-1108.

[5] Glosten, L.R. et al. 1993. On the relation between the expected value and the volatility of the Nominal Excess Return on Stocks. The Journal of Finance (48): 17791801.

[6] Nelson, R.B. 2006. An introduction to copulas, 2nd ed. New York: Springer.

[7] Officer Robert R. 1973. The variability of market factor of the New York stock exchange. Journal of Business (46): 434-453.

[8] Patton, A.J. 2001. Modeling time-varying exchange rate dependence using the conditional Copula. San Diego: Department of Economics University of California

[9] Patton, A.J. 2006. Modeling asymmetric exchange rate dependence. International Economic Review (47): 527556. 